Artigo Original

Original Article

Raul Philipe Marcos Silva ${ }^{1}$ (B) Cynthia Maria Barboza do Nascimento ${ }^{1}$ (1) Gabriella Morais Duarte Miranda² (B) Vanessa Lima da Silva ${ }^{3}$ Maria Luiza Lopes Timóteo de Lima 4 (C) Mirella Bezerra Rodrigues Vilela' ${ }^{1}$ (C)

Descritores

Oferta

Fonoaudiologia Acesso aos Serviços de Saúde Sistema Único de Saúde Indicadores Sociais

Keywords

Availability

Speech Therapy

Access to Health Services Unified Health System Social Indicators

Endereço para correspondência: Mirella Bezerra Rodrigues Vilela Departamento de Fonoaudiologia, Universidade Federal de Pernambuco UFPE

Rua Professor Artur de Sá, s/n, Cidade Universitária - Recife (PE) - Brasil CEP 50740-520.

E-mail: mirellarod@hotmail.com

Recebido em: Outubro 15, 2019

\section{Evolução da oferta de Fonoaudiólogos no SUS: um estudo sobre a correlação com os indicadores sociais no Brasil na última década}

\author{
Evolution of speech therapists supply in \\ SUS: a study about the correlation with social \\ indicatiors in Brazil in the last decade
}

\begin{abstract}
RESUMO
Objetivo: Investigar a correlação entre a oferta de Fonoaudiólogos no Sistema Único de Saúde (SUS) e os indicadores sociais no Brasil, entre 2007 e 2016. Método: Estudo ecológico, cujas unidades de análise foram as 27 Unidades Federativas (UFs) do país. Foram calculados os indicadores da oferta de Fonoaudiólogos no SUS e a evolução relativa desta oferta no período, bem como o Índice de Desenvolvimento Humano (IDH) e o Índice de Gini, ambos referentes ao ano de 2010. A correlação foi investigada utilizando o teste de Spearman, com $\alpha=5 \%$. Resultados: Em 2007, a oferta de Fonoaudiólogos no SUS foi de 3,55/10 ${ }^{5}$ habitantes, com o menor indicador no Amazonas e o maior no Mato Grosso do Sul. Em 2016, este indicador quase dobrou no país, com permanência do menor valor no Amazonas e o maior no Piauí. Ocorreu uma importante evolução relativa da oferta de Fonoaudiólogos no Brasil, neste período, com significativas variações entre as UFs. Houve correlação negativa entre a evolução relativa na última década e o IDH, e positiva com o Índice de Gini. Conclusão: A evolução da oferta de Fonoaudiólogos no SUS foi maior nas unidades federativas com menor desenvolvimento humano e maior concentração de renda. Não obstante, os resultados referentes a 2016 mostraram a manutenção do status quo, com a maior oferta naquelas unidades federativas com maior desenvolvimento humano.
\end{abstract}

\begin{abstract}
Purpose: Investigate the correlation between the provision of speech therapists in the Brazilian public healthcare system and social indicators between 2007 and 2016. Methods: An ecological study was conducted. The units of analysis were the 27 federative units of Brazil. Indicators of the provision of speech therapists in public healthcare and the relative evolution of this provision in the period as well as the Human Development Index and Gini Index related to the year 2010 were analyzed. Correlations were investigated using Spearman's test ( $\alpha=5 \%)$. Results: In 2007, the provision of speech therapists in the public healthcare system was $3.55 / 10^{5}$ residents, with the lowest indicator in the state of Amazonas and the highest in the state of Mato Grosso do Sul. In 2016, this indicator nearly doubled in the country; the lowest indicator was again in the state of Amazonas and the highest was in the state of Piauí. An important relative evolution occurred in the provision of speech therapists in Brazil in the period analyzed, with significant variation among the federative units. A negative correlation was found between the relative evolution in the last decade and the Human Development Index and a positive correlation was found with the Gini Index. Conclusion: The increase in the provision of speech therapists in the Brazilian public healthcare system was greater in federative units with lower human development and a greater concentration of income. Despite this, the results from 2016 showed the maintenance of the status quo, with a greater provision in federative units with greater human development.
\end{abstract}

\footnotetext{
Trabalho realizado na Universidade Federal de Pernambuco - UFPE, Recife (PE), Brasil.

' Departamento de Fonoaudiologia, Universidade Federal de Pernambuco - UFPE - Recife (PE), Brasil.

${ }^{2}$ Área Acadêmica de Medicina Social e Programa de Pós-graduação em Saúde Coletiva, Universidade Federal de Pernambuco - UFPE - Recife (PE), Brasil.

${ }^{3}$ Departamento de Fonoaudiologia e Programa de Pós-graduação em Gerontologia, Universidade Federal de Pernambuco - UFPE - Recife (PE), Brasil.

${ }^{4}$ Departamento de Fonoaudiologia e Programa de Pós-graduação em Saúde da comunicação Humana, Universidade Federal de Pernambuco - UFPE - Recife (PE), Brasil.
}

Fonte de financiamento: Nada a declarar.

Conflito de interesses: Nada a declarar 


\section{INTRODUÇÃO}

A Fonoaudiologia atua nos diferentes níveis de atenção à saúde, seja em ambulatórios, hospitais ou nos Núcleos Ampliados de Saúde da Família e Atenção Básica (NASF-AB) ${ }^{(1)}$. No que diz respeito à atuação na atenção primária, com a implantação do NASF, em 2008, houve aumento na oferta de procedimentos e práticas de cuidado à saúde no $\mathrm{SUS}^{(2,3)}$, favorecendo o acesso da população a este profissional ${ }^{(4)}$.

Ainda são poucos os estudos sobre a distribuição e oferta de profissionais de Fonoaudiologia no país. Três deles concluíram que a assistência fonoaudiológica no SUS acontece de forma desigual entre as macrorregiões e os estados brasileiros ${ }^{(2,3,5)}$.

As desigualdades, sejam de cunho social, regional ou étnico, constituem características marcantes na sociedade brasileira, que a aflige ao longo de toda a sua história ${ }^{()}$. Quando as desigualdades são consideradas injustas, evitáveis e desnecessárias, assumem uma conotação do princípio de justiça social e passam a ser denominadas iniquidades ${ }^{(7)}$, cujos aspectos socioeconômicos e ambientais assumem o protagonismo nesse cenário.

Dentre os indicadores que avaliam as iniquidades sociais inclui-se o IDH que, a despeito de ter crescido nos últimos anos, oscila entre os estados brasileiros ${ }^{(8)}$, e o Índice de Gini, um indicador de desigualdade segundo a renda domiciliar per capita que tem relação direta com as condições sociais e de saúde ${ }^{(9)}$. Evidências científicas apontam relação entre indicadores sociais, como o IDH e o Índice de Gini, e os indicadores de saúde geral da população ${ }^{(10-12)}$.

A equidade constitui um dos princípios doutrinários do SUS e, com base neste princípio, pesquisadores apontam relações entre o acesso e os fatores que permeiam a dinâmica social do país ${ }^{(13-16)}$.

Acesso é um termo de definição complexa, que tem motivado muitas produções científicas ${ }^{(14-17)}$. Nesse cenário, alguns autores defendem que $\mathrm{o}$ acesso pode ser compreendido na perspectiva do ingresso ou forma de entrada nos serviços de saúde e relacionar-se a características da oferta de serviços, referindo-se à possibilidade de utilizar serviços de saúde quando necessário( ${ }^{(17,18)}$. O uso efetivo dos serviços de saúde pode ser uma medida de avaliação do acesso, pois resulta de uma multiplicidade de fatores que incluem os individuais predisponentes, os contextuais e aqueles relativos à qualidade do cuidado, influenciando o uso e a efetividade do cuidado ${ }^{(17)}$.

Estudos mostram os avanços alcançados pelo SUS para assegurar um acesso universal ${ }^{(19,20)}$. No entanto, a despeito do progresso em relação à prestação de serviços, ainda são registradas disparidades no acesso aos serviços em saúde ${ }^{(14,15)}$ e desigualdades na distribuição de profissionais e serviços por região ${ }^{(5,6,14,15)}$, reconhecidos como importantes desafios para este sistema público ${ }^{(6)}$.

Diante desse cenário, surge a indagação referente às relações entre o acesso à Fonoaudiologia no Sistema Público de Saúde e os indicadores sociais no Brasil. Assim, este estudo teve por objetivo investigar a relação entre a oferta de Fonoaudiólogos no SUS e sua correlação com os indicadores sociais no Brasil, no período de 2007 a 2016.

\section{MÉTODOS}

$\mathrm{O}$ estudo se enquadra nos preceitos éticos previstos na resolução n 466/2012 do Conselho Nacional de Saúde e, por se basear em dados de domínio publico, não necessita de apreciação pelo Comitê de Ética em Pesquisa, sendo resguardado o dever de divulgar todas as fontes de dados.

Trata-se de um estudo ecológico, de base populacional, cujas unidades de análise foram as 27 UFs brasileiras, estudadas entre 2007 e 2016, última década com dados disponíveis nos sistemas de informação, durante o período da coleta de dados.

Foram consideradas variáveis dependentes o indicador da oferta de profissionais Fonoaudiólogos atuantes no SUS, aqui denominado Oferta de Fonoaudiologia no SUS, em 2016, e a evolução relativa dessa oferta durante o período estudado (20072016). Os métodos de cálculo estão descritos nas fórmulas abaixo:

$$
\text { Oferta de Fonoaudiologia no SUS }=\frac{\begin{array}{c}
\mathrm{N}^{\mathrm{o}} \text { de Fonoaudiólogos no } \\
\text { SUS, no ano } \\
\text { na UF }
\end{array}}{\begin{array}{c}
\text { Total de habitantes no } \\
\text { ano }_{\mathrm{x}} \text { na UF }_{\mathrm{x}}
\end{array}} \mathbf{X} 10^{5}
$$

Evolução relativa daoferta $=\frac{\left[\left(\begin{array}{c}\text { Oferta de Fonoaudiólogo } \\ \text { no SUS, em } 2016\end{array}\right)-1\right]}{\text { Oferta de Fonoaudiólogo }}$ X100

O número de profissionais Fonoaudiólogos atuantes no SUS foi coletado no banco de dados do Cadastro Nacional de Estabelecimentos de Saúde (CNES), utilizando os arquivos com extensão *.dbc do mês de dezembro de cada ano, disponibilizados pelo site do Departamento de Informática do Ministério da Saúde e processados pelo software Tabwin, versão 3.2. Para as informações referentes aos habitantes de cada UF foram utilizadas as estimativas censitárias dos anos de 2007 e 2016. Os dados do IDH e do Índice de Gini são referentes ao ano de 2010, publicados no Atlas do Desenvolvimento Humano, $2013^{(9)}$. O ano-base de 2010 foi selecionado, por tratar-se de ano censitário no país.

Ambos os indicadores sociais têm valores que variam de zero a um. No entanto, suas interpretações são opostas. Para o IDH, quanto maior o valor, melhor o nível de desenvolvimento humano. Para o Índice de Gini, quanto mais próximo de um, maior é a concentração de renda, portanto, maior a desigualdade.

Os dados foram analisados por meio de estatística descritiva, utilizando as medidas de tendência central e dispersão. Foi testada a normalidade da distribuição das variáveis dependentes por meio do teste de Shapiro-Wilk, $\operatorname{com} \alpha=5 \%$. Como a distribuição foi não normal, utilizou-se o teste de Sperman para investigar a correlação entre as variáveis dependentes e independentes, também com $\alpha=5 \%$.

Foram elaborados mapas temáticos com a distribuição das variáveis independentes e dos indicadores sociais, utilizandose a distribuição por quartis, através do programa Terraview, 
versão 4.2.1, desenvolvido pelo Instituto Nacional de Pesquisas Espaciais (INPE).

\section{RESULTADOS}

A oferta de Fonoaudiologia no SUS, em 2007, foi de 3,55/10 habitantes, variando de $0,68 / 10^{5}$, no Amazonas, a 5,64/10 , no Paraná $(\delta=1,38 ; \mathrm{Md}=2,79)$. No ano de 2016, a oferta foi de $6,18 / 10^{5}$ habitantes, variando de $2,57 / 10^{5}$, também no Amazonas, a 7,69/10 habitantes, no Amapá $(\delta=1,61 ; \mathrm{Md}=6,13)$ (Tabela 1 , Figuras 1A e 1B).

A evolução relativa mostrou um incremento de 74,26\% na oferta de Fonoaudiologia no SUS na última década, com aumento também em todas as UFs. Houve variação de 36,11\%, no Paraná, e 505,29\%, em Sergipe ( $\delta=115,6$; Md=91,4) (Tabela 1 e Figura 1C).

Em relação aos indicadores sociais no Brasil, o Índice de Gini foi de 0,59, variando de 0,49, em Santa Catarina, a 0,65, no Amazonas $(\delta=0,039 ; \mathrm{Md}=0,6)$ (Tabela 2 e Figura $2 \mathrm{~B})$. $\mathrm{O}$ IDH do Brasil foi de 0,7, variando de 0,63, em Alagoas, a 0,82, no Distrito Federal $(\delta=0,05 ; \mathrm{Md}=0,7)$ (Tabela 2 e Figura $2 \mathrm{~A})$. O IDHME (educação) do Brasil foi de 0,6, variando de 0,52, em Alagoas, a 0,74 , no Distrito Federal $(\delta=0,06 ; \mathrm{Md}=0,6)$. O
IDHML (longevidade) do Brasil foi de 0,8, variando de 0,75, em Alagoas, a 0,87 , no Distrito Federal $(\delta=0,03 ; \mathrm{Md}=0,8)$. O IDHMR (renda) do Brasil foi de 0,7, variando de 0,61, no Maranhão, a $0,86$, no Distrito Federal $(\delta=0,05 ; \mathrm{Md}=0,7)$ (Tabela 2$)$.

Houve correlação positiva entre a oferta de Fonoaudiologia em 2016 e o IDH entre as UFs ( $r s=0,37 ; p=0,05)$ e correlação negativa entre esta oferta e o Índice de Gini ( $r s=-0,49 ; p=0,01)$ (Tabela 3).

A análise da evolução relativa, na última década, entre as UFs, evidenciou correlação negativa com o IDH ( $\mathrm{rs}=-0,54 ; \mathrm{p}=0,004)$ e positiva com o Índice de Gini ( $\mathrm{rs}=0,62 ; \mathrm{p}=0,0006)$ (Tabela 3 ).

A relação entre o IDH renda, IDH longevidade e IDH escolaridade, e as variáveis dependentes seguiu o mesmo padrão observado no IDH, mantendo a significância estatística apenas no componente IDH escolaridade (Tabela 3).

A distribuição espacial dos indicadores da oferta de Fonoaudiologia no SUS em 2007 e 2016 evidenciou maior concentração nas UFs localizadas na Região Sudeste. Essa distribuição apresentou comportamento distinto quando observada a evolução da oferta, com maior ampliação naquelas situadas nas regiões Norte e Nordeste (Figura 1A e Figura 1B).

A distribuição espacial dos indicadores sociais evidenciou comportamento espelhado entre o IDH e Gini. As UFs em

Tabela 1. Oferta de Fonoaudiologia no SUS e sua evolução relativa, segundo as Unidades da Federação, Brasil, 2007 e 2016

\begin{tabular}{|c|c|c|c|}
\hline Unidade da Federação & Oferta de Fonoaudiologia SUS 2007 & Oferta de Fonoaudiologia SUS 2016 & Evolução relativa \\
\hline Rondônia & 2,96 & 4,03 & 36,20 \\
\hline Acre & 2,59 & 4,04 & 55,78 \\
\hline Amazonas & 0,68 & 2,57 & 276,96 \\
\hline Roraima & 2,78 & 6,42 & 130,87 \\
\hline Pará & 1,10 & 3,05 & 176,91 \\
\hline Amapá & 2,38 & 7,67 & 221,75 \\
\hline Tocantins & 4,34 & 6,59 & 51,74 \\
\hline Maranhão & 0,93 & 4,10 & 339,96 \\
\hline Piauí & 1,68 & 7,69 & 357,21 \\
\hline Ceará & 2,39 & 4,51 & 88,22 \\
\hline Rio Grande do Norte & 3,82 & 7,05 & 84,76 \\
\hline Paraíba & 3,32 & 7,58 & 128,00 \\
\hline Pernambuco & 2,79 & 5,35 & 91,40 \\
\hline Alagoas & 2,60 & 6,13 & 135,78 \\
\hline Sergipe & 0,72 & 4,37 & 505,29 \\
\hline Bahia & 1,26 & 3,50 & 178,60 \\
\hline Minas Gerais & 4,56 & 7,34 & 60,92 \\
\hline Espírito Santo & 3,25 & 5,03 & 54,76 \\
\hline Rio de Janeiro & 4,87 & 7,44 & 52,68 \\
\hline São Paulo & 5,00 & 7,49 & 49,68 \\
\hline Paraná & 5,64 & 7,68 & 36,11 \\
\hline Santa Catarina & 3,34 & 7,48 & 123,92 \\
\hline Rio Grande do Sul & 2,65 & 5,80 & 119,35 \\
\hline Mato Grosso do Sul & 4,94 & 6,86 & 38,77 \\
\hline Mato Grosso & 3,33 & 5,05 & 51,81 \\
\hline Goiás & 3,68 & 6,15 & 67,05 \\
\hline Distrito Federal & 1,67 & 4,57 & 173,63 \\
\hline Total & 3,55 & 6,18 & 74,26 \\
\hline
\end{tabular}

Legenda: Os valores da oferta de Fonoaudiologia em 2007 e 2016 são considerados para cada 100 mil habitantes. A evolução relativa é dada em forma de percentual

Fonte: Cadastro Nacional de Estabelecimentos de Saúde (CNES) do Ministério da Saúde ${ }^{(21)}$ 


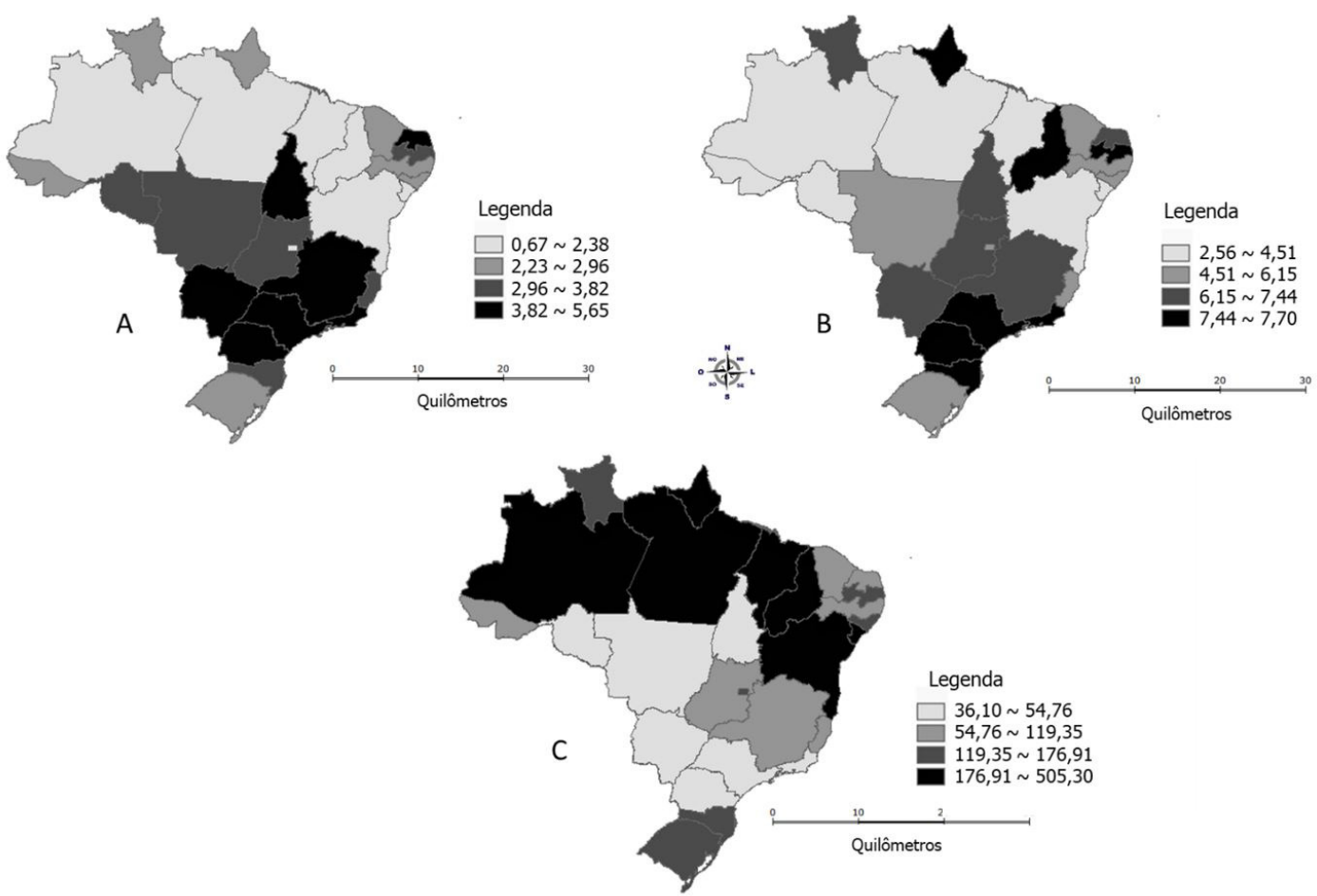

Legenda: (A) Coeficiente de oferta de Fonoaudiólogo no SUS, 2007; (B) Coeficiente de oferta de Fonoaudiólogo no SUS, 2016; (C) Coeficiente de evolução relativa da oferta de Fonoaudiólogo no SUS, 2007-2016

Figura 1. Distribuição espacial da oferta de Fonoaudiologia no SUS e sua evolução relativa, segundo as Unidades da Federação, Brasil, 2007 e 2016
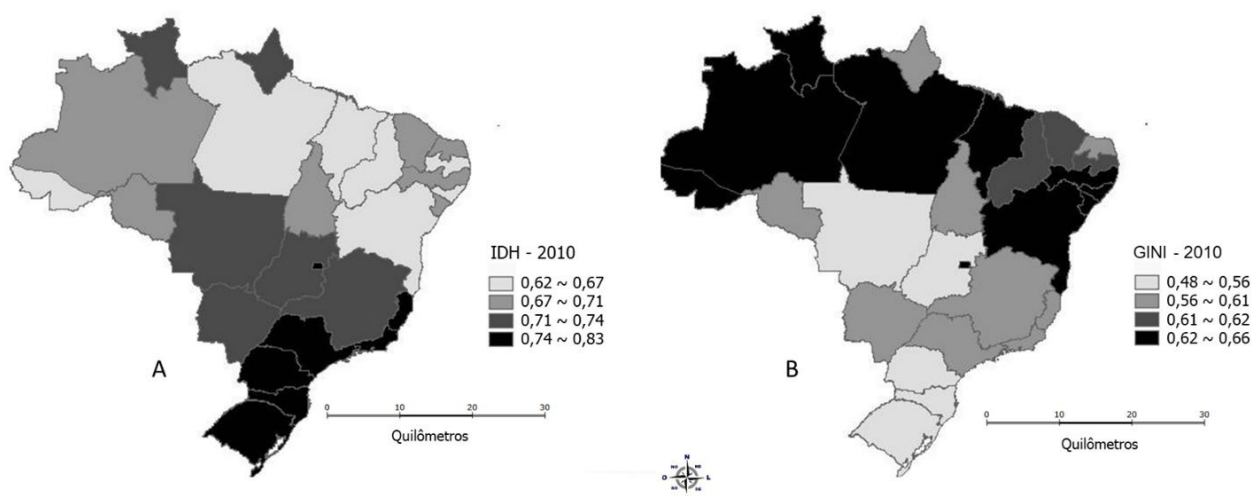

Legenda: (A) Índice de Desenvolvimento Humano, 2010; (B) Índice de Gini, 2010.

Figura 2. Distribuição espacial do Índice de Desenvolvimento Humano e Índice de Gini, segundo as unidades da Federação, Brasil, 2010

que o IDH apontou para maior desenvolvimento humano, mais escuras no mapa, são aquelas em que o Índice de Gini apresentou menores valores, indicando menor concentração de renda (Figura 2A e Figura 2B).

\section{DISCUSSÃO}

Os resultados encontrados neste estudo apontam correlação entre os indicadores sociais e a oferta de Fonoaudiologia no SUS.

Para compreender melhor os resultados, é importante destacar que existe uma relação inversamente proporcional entre IDH e Gini. À medida que um aumenta, o outro diminui, evidenciando que a concentração de renda tem repercussões diretas sobre o desenvolvimento humano de uma localidade ${ }^{(22)}$.

Tanto em 2007 como em 2016 os maiores indicadores de oferta de Fonoaudiologia foram encontrados nas UFs das regiões Sul e Sudeste. Nessas regiões também foram encontrados os maiores índices de IDH e os menores de Gini, evidenciando que as UFs que mais ofertam esse profissional no SUS apresentam maior nível de desenvolvimento humano e, portanto, menor concentração de renda. De forma semelhante, os menores indicadores de oferta de Fonoaudiologia em ambos os anos foram encontrados nas UFs das regiões Norte e Nordeste, as quais apresentaram 
Tabela 2. Índice de Gini, IDHM, IDHME, IDHML e IDHMR, segundo as Unidades da Federação, Brasil, 2010

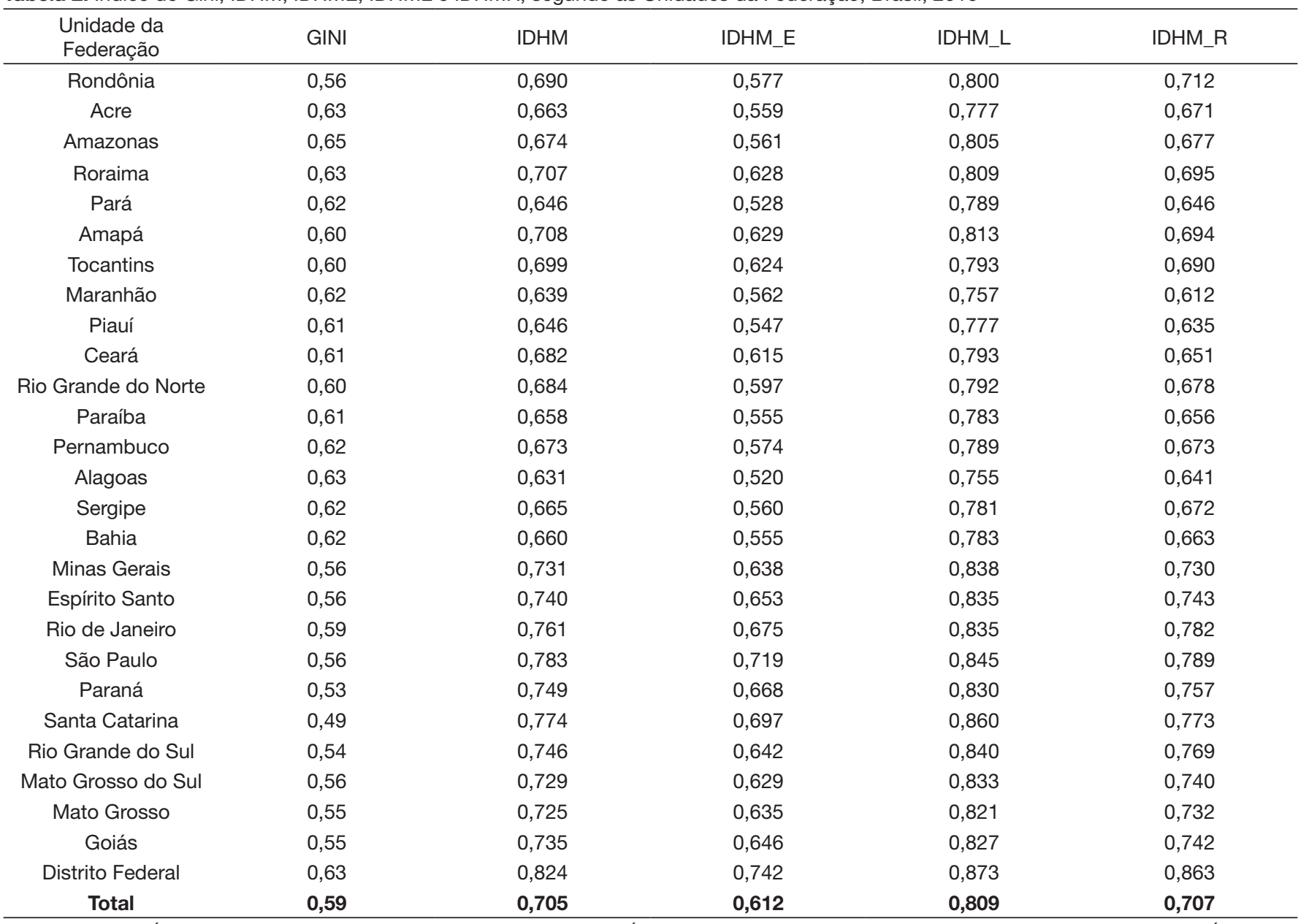

Legenda: IDHM (Índice de Desenvolvimento Humano Municipal), IDHME (Índice de Desenvolvimento Humano Municipal - Educação), IDHML (Índice de Desenvolvimento Humano Municipal - Longevidade), IDHMR (Índice de Desenvolvimento Humano Municipal - Renda)

Fonte: Atlas do Desenvolvimento Humano(9)

Tabela 3. Coeficiente de correlação de spearman entre os indicadores sociais e as variáveis dependentes

\begin{tabular}{ccccccc}
\hline \multirow{2}{*}{ Indicadores Sociais } & \multicolumn{2}{c}{ Oferta de Fonoaudiologia no SUS em 2016 } & & \multicolumn{3}{c}{ Evolução relativa 2007-2016 } \\
\cline { 2 - 3 } \cline { 5 - 6 } & Coeficiente de Spearman & $\mathrm{p}$-valor & & Coeficiente de Spearman & $\mathrm{p}$-valor \\
\hline IDH & $\mathrm{rs}=0,37$ & $\mathrm{p}=0,056$ & $\mathrm{p}=0,051$ & $\mathrm{rs}=-0,54$ & $\mathrm{p}=0,004$ \\
IDHE & $\mathrm{rs}=0,38$ & & $\mathrm{rs}=-0,51$ & $\mathrm{p}=0,007$ \\
IDHL & $\mathrm{rs}=0,31$ & $\mathrm{p}=0,122$ & & $\mathrm{rs}=-0,45$ & $\mathrm{p}=0,018$ \\
IDHR & $\mathrm{rs}=0,32$ & $\mathrm{p}=0,110$ & & $\mathrm{rs}=-0,55$ & $\mathrm{p}=0,003$ \\
Índice de Gini & $\mathrm{rs}=-0,49$ & $\mathrm{p}=0,01$ & & $\mathrm{rs}=0,62$ & $\mathrm{p}=0,0006$ \\
\hline
\end{tabular}

Legenda: IDH (Índice de Desenvolvimento Humano), IDHE (Índice de Desenvolvimento Humano- Educação), IDHL (índice de Desenvolvimento Humano Longevidade), IDHR (Índice de Desenvolvimento Humano - Renda)

menores índices de IDH e maiores de Gini, revelando que a maior dificuldade no acesso a este profissional no SUS está nas UFs localizadas em regiões com maior concentração de renda e, portanto, menor desenvolvimento humano. Esses achados apontam para uma desigualdade persistente, fato corroborado em estudos anteriores ${ }^{(2,3,5,23,24)}$.

Esses estudos evidenciaram que os maiores percentuais de procedimentos e de profissionais Fonoaudiólogos no SUS também se concentravam em áreas com condições socioeconômicas mais favoráveis, como as regiões Sudeste e Sul, e os menores em áreas de piores condições socioeconômicas, como as regiões Norte e Nordeste ${ }^{(2,5,23,24)}$.

Estudo sobre a assistência fonoaudiológica no SUS observou que a região Sudeste, a mais rica do Brasil, concentrava, no ano de 2010, mais da metade dos procedimentos registrados no país e dispunha do maior percentual de profissionais Fonoaudiólogos . Observou também a disparidade entre a quantidade e a necessidade de profissionais Fonoaudiólogos no SUS, nas regiões Norte e Nordeste, evidenciando um déficit nessas regiões no período estudado $^{(5)}$. 
De forma semelhante, estudos que analisaram a evolução da oferta de Fonoaudiólogos no SUS e na APS no Brasil, entre 2008 e $2013^{(2)}$ e 2005 e $2015^{(3)}$ constataram maior concentração de oferta na Região Sudeste e menor na Região Norte, evidenciando que a distribuição de Fonoaudiólogos no território nacional parece manter relações com as desigualdades sociais.

Quanto a este mesmo aspecto - desigualdades intrarregionais -, estudo observou distribuição insuficiente e desigual na oferta de Fonoaudiólogos nos serviços de saúde pública municipais entre as capitais da Região Nordeste do Brasil, comprometendo 0 acesso da população a esses serviços ${ }^{(24)}$.

Esse achado não é apenas para a área da Fonoaudiologia. Pesquisa sobre os indicadores de atenção básica em saúde bucal e sua relação com condições socioeconômicas ${ }^{(25)}$ concluiu que os municípios com maior IDH no Brasil foram os que obtiveram as maiores proporções de primeira consulta odontológica, fato que pode ser explicado pela melhor provisão de serviços que esses locais fornecem à população. Esse mesmo estudo ${ }^{(25)}$ identificou também que os estados do Rio Grande do Sul e Santa Catarina, integrantes da Região Sul, apresentaram as maiores proporções de profissionais cirurgiões-dentistas nos serviços de saúde.

No que se refere à evolução relativa da oferta de Fonoaudiologia no período estudado, houve expansão em todas as UFs. No entanto, os maiores índices desta evolução foram encontrados nas UFs das regiões Norte e Nordeste, as quais obtiveram os menores índices de IDH e os maiores de Gini. Já os menores índices de evolução foram encontrados em UFs das regiões Sudeste, Sul e Centro-Oeste: os maiores índices de IDH e os menores de Gini.

Resultados semelhantes foram constatados em estudo que observou a evolução da oferta de Fonoaudiologia no SUS em todas as UFs do Brasil, especialmente nos estados de Sergipe, Piauí e Bahia, todos da Região Nordeste ${ }^{(2)}$. Houve também expansão do quantitativo de profissionais Fonoaudiólogos em todas as regiões do Brasil, entre os anos de 2000 e 2010, destacando-se a Região Norte, e, entre os anos de 2007 e 2014, houve aumento dessa oferta nas capitais da Região Nordeste ${ }^{(5,24)}$. No período de 2005 a 2015 observou-se crescimento superior ao dobro em número de Fonoaudiólogos no nível primário de Atenção à Saúde no $\mathrm{SUS}^{(3)}$, com maior concentração também na Região Sudeste.

Entretanto, a despeito da evolução relativa da última década mostrar maior expansão no acesso à Fonoaudiologia no SUS para aquelas UFs com menor desenvolvimento humano e maior concentração de renda, os resultados encontrados em 2016 demonstram poucas mudanças no padrão encontrado em 2007, evidenciando que ainda há caminhos a ser percorridos para assegurar uma oferta equânime.

A evolução da oferta de Fonoaudiologia no SUS, constatada neste estudo, não foi suficiente para contemplar a demanda existente naquelas UFs com baixos níveis de desenvolvimento humano. Alguns autores concordam que houve redução dessas desigualdades, especialmente nos últimos anos ${ }^{(2,5,23,24,26,27)}$. No entanto, percebe-se que ainda há disparidades na oferta de Fonoaudiólogos e desigualdades na sua distribuição entre as UFs, em que as regiões com melhores condições socioeconômicas detêm os melhores indicadores de oferta de Fonoaudiologia.
Por sua vez, as que apresentam piores indicadores sociais têm menor oferta, semelhante ao encontrado em outros $\operatorname{estudos}^{(5,7,26,27)}$.

Acredita-se que a expansão do acesso ao profissional fonoaudiólogo nas regiões com mais baixas condições socioeconômicas do país, Norte e Nordeste, certamente está relacionada à ampliação da cobertura da atenção básica nessas regiões, orientada sobretudo pela Equidade, princípio norteador do SUS. Quanto às regiões Sul e Sudeste, apesar de concentrarem as UFs com os melhores indicadores de oferta, trabalho ${ }^{(3)}$ que analisou essa oferta na atenção básica mensurou um déficit de $65,4 \%$ e $42,3 \%$ no quantitativo de profissionais necessários, respectivamente. Isso evidencia que, a despeito das desigualdades entre as regiões brasileiras, a necessidade de ampliação do acesso à Fonoaudiologia é para todo o território nacional.

Os autores reconhecem algumas limitações metodológicas do estudo- o fato de utilizar dados secundários, sujeitos a subnotificação e qualidade das informações, e o nível de agregação estadual, sujeito a flutuações devido ao uso do valor médio do IDH e Gini. Não obstante, destaca-se que esses dados dos sistemas de informações oficiais do Ministério da Saúde, como os do CNES, e de plataformas oficiais da Organização das Nações Unidas (ONU), no caso dos indicadores sociais são importantes para a realização de estudos acadêmicos e a produção de evidências que contribuem para o planejamento, implantação e aprimoramento de serviços e ações inerentes à saúde pública. Registra-se também que não foi investigada a migração de Fonoaudiólogos de uma unidade federativa para outra. Os autores acreditam que essas migrações são pontuais e certamente não causariam alterações importantes nos resultados encontrados.

O estudo com dados secundários possibilitou a elaboração de hipóteses que podem ser confirmadas mediante a realização de novos estudos que contemplem essa problemática, com o objetivo de produzir e discutir informações que contribuam para a consolidação dos serviços de Fonoaudiologia no SUS e, por sua vez, para a melhoria do acesso à saúde, respeitando os princípios de universalidade e equidade no SUS.

\section{CONCLUSÃO}

A evolução da oferta de Fonoaudiologia foi maior nas unidades federativas com menor desenvolvimento humano e maior concentração de renda. Esse achado pode revelar que a forma como ocorreu esta expansão na última década foi uma tentativa de minimizar os efeitos da desigualdade social no acesso à saúde da comunicação humana no SUS. Não obstante, os resultados referentes a 2016 evidenciaram a manutenção do status quo, com maior oferta de Fonoaudiologia naquelas unidades federativas com maior desenvolvimento humano.

É preciso envidar todos os esforços para que o caminho de mudanças não seja perdido, uma vez que os avanços alcançados ainda não superam a pouca oferta da assistência fonoaudiológica em todo o país. Um país tão desigual, que optou pela universalidade do acesso, precisa assegurar que o cuidado esteja ao alcance de todos que dele precisam. E com a Fonoaudiologia não pode ser diferente: ela deve ser direito de todos e dever do Estado. 


\section{REFERÊNCIAS}

1. Molini-Avejonas DR, Aboboreira MS, Couto MIV, Samelli AG. Insertion and performance of Speech-Language Pathology and Audiology in Family Health Support Centers. CoDAS. 2014;26(2):148-54. http://dx.doi. org/10.1590/2317-1782/2014011IN. PMid:24918508.

2. Sousa MFS, Nascimento CMB, Sousa FOS, Lima MLLT, Silva VL, Rodrigues M. Evolução da oferta de fonoaudiólogos no SUS e na atenção primária à saúde, no Brasil. Rev CEFAC. 2017;19(2):213-20. http://dx.doi. org/10.1590/1982-0216201719215816.

3. Viégas LHT, Meira TC, Santos BS, Mise YF, Arce VAR, Ferrite S. Speech, Language and Hearing services in Primary Health Care in Brazil: an analysis of provision and an estimate of shortage, 2005-2015. Rev CEFAC. 2018;20(3):353-62. http://dx.doi.org/10.1590/1982-021620182031918.

4. Ferreira CL, Silva FR, Martins-Reis VO, Friche AAL, Santos JN. Distribuição dos fonoaudiólogos na atenção à saúde no estado de Minas Gerais entre 2005 e 2010. Rev CEFAC. 2013;15(3):672-80. http://dx.doi. org/10.1590/S1516-18462013005000011.

5. Miranda GMD, Mendes ACG, Silva ALA, Rodrigues M. Assistência fonoaudiológica no sus: a ampliação do acesso e o desafio de superação das desigualdades. Rev CEFAC. 2015;17(1):71-9. http://dx.doi.org/10.1590/19820216201515213 .

6. Victora CG, Barreto ML, Leal MC, Monteiro CA, Schmidt MI, Paim, JS, et al. Condições de saúde e inovações nas políticas de saúde no Brasil: o caminho a percorrer. Saúde no Brasil. 2011;6:90-102.

7. Whitehead M. The concepts and principles of equity and health. Int $\mathrm{J}$ Health Serv. 1992;22(3):429-45. http://dx.doi.org/10.2190/986L-LHQ62VTE-YRRN. PMid:1644507.

8. PNUD: Programa das Nações Unidas para o Desenvolvimento. Relatório do Desenvolvimento Humano 2010. Nova Iorque: PNUD; 2010.

9. PNUD: Programa das Nações Unidas para o Desenvolvimento. Atlas do Desenvolvimento Humano no Brasil 2013. Nova Iorque: PNUD; 2013.

10. Chavehpour Y, Rashidian A, Woldemichael A, Takian A. Inequality in geographical distribution of hospitals and hospital beds in densely populated metropolitan cities of Iran. BMC Health Serv Res. 2019;19(1):614. http:// dx.doi.org/10.1186/s12913-019-4443-0. PMid:31470849.

11. Xiong J, Ye C, Zhou T, Cheng W. Health risk and resilience assessment with respect to the main air pollutants in Sichuan. Int J Environ Res Public Health. 2019;16:2796. http://dx.doi.org/10.3390/ijerph16152796.

12. Okhovat-Isfahani B, Bitaraf S, Ali Mansournia M, Doosti-Irani A. Inequality in the global incidence and prevalence of tuberculosis (TB) and TB/HIV according to the human development index. Med J Islam Repub Iran. 2019;22(33):45. http://dx.doi.org/ 10.34171/mjiri.33.45.

13. Andrade KVF, Nery JS, Pescarini JM, Ramond A, Santos CAST, Ichihara MY, et al. Geographic and socioeconomic factors associated with leprosy treatment default: an analysis from the 100 Million Brazilian Cohort. PLoS Negl Trop Dis. 2020;14(9):e0008723. http://dx.doi.org/10.1371/journal. pntd.0007714.

14. Silva MELE, Almeida ATC, Araújo IT Jr. Equity analysis of resource distribution for the Popular Pharmacy Program. Rev Saude Publica. 2019;20(53):50. http://dx.doi.org/10.11606/S1518-8787.2019053000731. PMid:31116239.
15. Gómez EJ, Jungmann S, Lima AS. Resource allocations and disparities in the Brazilian health care system: insights from organ transplantation services. BMC Health Serv Res. 2018;18(1):90. http://dx.doi.org/10.1186/ s12913-018-2851-1. PMid:29415705.

16. Beenackers MA, Goldbaum M, Barros MBA, Gianini RJ, Cesar CLG, Mackenbach JP. Use, access, and equity in health care services in São Paulo, Brazil. Cad Saude Publica. 2017;33(4):e00078015. http://dx.doi. org/10.1590/0102-311X00078015. PMid:28538789.

17. Travassos C, Martins M. Uma revisão sobre os conceitos de acesso e utilização de serviços de saúde. Cad. Saúde Pública. 2004;20(2):190-8.

18. Barbosa SP, Elizeu TS, Penna CMM. Ótica dos profissionais de saúde sobre o acesso à atenção primária à saúde. Ciênc. saúde Coletiva. 2013;18(2):34757.

19. Paim J, Travassos C, Almeida C, Bahia L, Macinko J. The Brazilian health system: history, advances, and challenges. Lancet. 2011;377(9779):177897. PMid:21561655.

20. Assis MMA, Jesus WLA. Acesso aos serviços de saúde: abordagens, conceitos, políticas e modelo de análise. Ciênc. saúde Coletiva. 2012;17(11):2865-75.

21. Brasil. Ministério da Saúde. Cadastro Nacional de Estabelecimentos de Saúde (CNES). Brasília: Ministério da Saúde; 2017.

22. Sálvio GMM, Fontes MAL, Silveira WJ Jr, Silva HA. Áreas de conservação, pobreza e desigualdade social: avaliação utilizando indicadores socioeconômicos em minas gerais, Brasil. Cerne. 2016;22(2):145-50. http://dx.doi.org/10.1 590/01047760201622022151

23. Santos JN, Maciel FJ, Martins VO, Rodrigues ALV, Gonzaga AF, Silva LF. Inserção dos fonoaudiólogos no SUS/MG e sua distribuição no território do estado de Minas Gerais. Rev CEFAC. 2011;14(2):196-205. http://dx.doi org/10.1590/S1516-18462011005000088.

24. Santos JAP, Arce VAR, Magno LD, Ferrite S. Provision of Speech, Language and Hearing services in the public municipal healthcare network in the state capitals of Northeast Brazil. Audiol Commun Res. 2017;22:e1665.

25. Fischer TK, Peres KG, Kupek E, Peres MA. Indicadores de atenção básica em saúde bucal: associação com as condições socioeconômicas, provisão de serviços, fluoretação de águas e a estratégia de saúde da família no Sul do Brasil. Rev Bras Epidemiol. 2010;13(1):126-38. http://dx.doi.org/10.1590/ S1415-790X2010000100012. PMid:20683561.

26. Almeida G, Sarti FM, Ferreira FF, Diaz MD, Campino AC. Analysis of the evolution and determinants of income-related inequalities in the brazilian health system, 1998-2008. Rev Panam Salud Publica. 2013;33(2):90-7, 4 , 90. http://dx.doi.org/10.1590/S1020-49892013000200003. PMid:23525338.

27. Macinko J, Lima-costa MF. Horizontal equity in health care utilization in brazil, 1998 - 2008. Int J Equity Health. 2012;11(33):1-8. http://dx.doi. org/10.1186/1475-9276-11-33. PMid:22720869.

\section{Contribuição dos autores}

RPMS participou das etapas de planejamento, coleta, análise, escrita e revisão final do manuscrito; CMBN, GMDM, VLM, MLLTL participaram das etapas de análise, escrita e revisão final do manuscrito, e MBRV participou na condição de orientadora, da idealização do estudo, análise, escrita e revisão final do manuscrito. 
Raul Philipe Marcos Silva ${ }^{1}$ Cynthia Maria Barboza do Nascimento ${ }^{1}$ (16)

Gabriella Morais Duarte Miranda² Vanessa Lima da Silva ${ }^{3}$ Maria Luiza Lopes Timóteo de Lima4 ${ }^{4}$

Mirella Bezerra Rodrigues Vilela ${ }^{1}$

\section{ERRATA: "Evolução da oferta de Fonoaudiólogos no SUS: um estudo sobre a correlação com os indicadores sociais no Brasil na última década"}

No artigo intitulado "Evolução da oferta de Fonoaudiólogos no SUS: um estudo sobre a correlação com os indicadores sociais no Brasil na última década", com o número do DOI: https://doi.org/10.1590/2317-1782/20202019243, publicado na revista CoDAS, 2021, volume 33, número 2, na página 1:

Onde se lia:

"Vanessa Lima da Silva ${ }^{3}$ http://orcid.org/0000-0002-0548-9664

Leia-se:

"Vanessa de Lima Silva ${ }^{3}$ http://orcid.org/0000-0002-1562-1761

\footnotetext{
${ }^{1}$ Departamento de Fonoaudiologia, Universidade Federal de Pernambuco - UFPE - Recife (PE), Brasil.

${ }^{2}$ Área Acadêmica de Medicina Social e Programa de Pós-graduação em Saúde Coletiva, Universidade Federal de Pernambuco - UFPE - Recife (PE), Brasil.

${ }^{3}$ Departamento de Fonoaudiologia e Programa de Pós-graduação em Gerontologia, Universidade Federal de Pernambuco - UFPE - Recife (PE), Brasil.

${ }^{4}$ Departamento de Fonoaudiologia e Programa de Pós-graduação em Saúde da comunicação Humana, Universidade Federal de Pernambuco - UFPE - Recife (PE), Brasil.
} 